\title{
Depression in patients with chronic hepatitis B and cirrhosis is closely associated with the severity of liver cirrhosis
}

\author{
HAI-PENG ZHU ${ }^{1}$, YU-RONG GU ${ }^{2}$, GENG-LIN ZHANG ${ }^{2}$, YU-JIE SU ${ }^{2}$, \\ $\mathrm{KE} \mathrm{WANG}^{2}$, YU-BAO ZHENG ${ }^{2}$ and ZHI-LIANG GAO ${ }^{2}$ \\ ${ }^{1}$ Department of Infectious Diseases, Dongguan People's Hospital, Dongguan, Guangdong 523059; \\ ${ }^{2}$ Department of Infectious Diseases, The Third Affiliated Hospital of Sun Yat-Sen University, \\ Guangzhou, Guangdong 510630, P.R. China
}

Received November 9, 2014; Accepted April 1, 2016

DOI: $10.3892 /$ etm.2016.3271

\begin{abstract}
Depression in patients with chronic hepatitis B (CHB) can affect the quality of life, disease diagnosis and case fatality rate. The aim of this study was to explore depression in patients with $\mathrm{CHB}$ and cirrhosis, and the effect of the severity of liver cirrhosis on the depressive emotional state. The depressive emotional state was investigated using the Hamilton Depression Scale (HAMD) and Hamilton Anxiety Scale (HAMA) in 114 patients with CHB and cirrhosis, comprising 42 cases classified as Child-Pugh grade (CPG)-A, 38 cases classified as CPG-B and 34 cases classified as $\mathrm{CPG}-\mathrm{C}$ at a single hepatology center. Patients with mood disorders accounted for $33.33 \%$ of the 114 cases with $\mathrm{CHB}$ and liver cirrhosis and comprised 10 cases of CPG-A, 12 cases of CPG-B and 16 cases of CPG-C classification. The results shows that HAMA and HAMD scores of patients in the CPG-C group were significantly higher than those in the CPG-A group $(\mathrm{P}<0.01)$, but not significantly higher than those in the $\mathrm{CPG}-\mathrm{B}$ group $(\mathrm{P}>0.05)$. The incidence rate of mood disorders in the CPG-C group was significantly higher than that in the $C P G-B$ group $(P=0.0336)$, and the incidence rate of mood disorders was higher in the $\mathrm{CPG}-\mathrm{B}$ group compared with the CPG-A group, but the difference was not statistically significant $(\mathrm{P}=0.4370)$. The incidence rate of mood disorders in patients in the CPG-A group was significantly lower than that in the CPG-C group ( $\mathrm{P}=0.0078)$. The study shows that a considerable proportion of patients with liver cirrhosis have mood disorders, and the depression rates of $\mathrm{CHB}$-infected patients with liver cirrhosis are closely associated with the severity of the cirrhosis.
\end{abstract}

Correspondence to: Professor Yu-Bao Zheng, Department of Infectious Diseases, The Third Affiliated Hospital of Sun Yat-Sen University, 600 Tianhe Road, Guangzhou, Guangdong 510630, P.R. China

E-mail: guangzhouzyb@126.com

Key words: hepatitis B, liver cirrhosis, depression, severity, Child-Pugh classification

\section{Introduction}

There are an estimated 400 million people with chronic hepatitis $\mathrm{B}$ (CHB) worldwide, and $\sim 8$ million patients succumb annually to severe $\mathrm{CHB}$, hepatocellular carcinoma and end-stage liver cirrhosis (1). End-stage liver cirrhosis is one of the main causes of mortality in CHB patients $(1,2)$. It is widely recognized that psychological factors influence the development and prognosis of cirrhosis in patients with CHB. The repeated outbreaks and protracted course of hepatitis $\mathrm{B}$ and liver cirrhosis result in different degrees of mood disorders in some patients. The incidence rate of negative emotions in patients with chronic hepatitis has been reported to be $38.1-51.1 \%$, among which $47 \%$ of patients have anxiety problems and $68 \%$ of patients have mild-to-moderate depression $(3,4)$. When depression occurs in combination with liver disease, it can affect the patient's quality of life, the disease diagnosis and case fatality rate (4). Patients with end-stage liver disease have committed suicide because of depression (4). The accurate proportion of patients with hepatitis B and cirrhosis suffering from depression is unclear; therefore, the evaluation of depression in such patients is very important. For this reason, the present study assessed the emotional state of 114 patients with hepatitis B and cirrhosis using the Hamilton Depression Scale (HAMD) and Hamilton Anxiety Scale (HAMA) to explore the effects of the severity of liver cirrhosis on the depressive emotional state.

\section{Patients and methods}

Patients. A prospective cohort that included 114 hospitalized patients (95 men and 19 women; mean age, $42.5 \pm 11.6$ years) with hepatitis B-associated liver cirrhosis was recruited from the Department of Infectious Diseases of the Third Affiliated Hospital of Sun Yat-Sen University (Guangzhou, China) between July 25, 2011 and December 15, 2013. The patients were divided into three groups by the Child-Pugh classification standard (5) to evaluate the severity of illness. These were the Child-Pugh grade (CPG)-A group including 42 patients (35 men and 7 women), CPG-B group including 38 patients ( 32 men and 6 women) and $C P G-C$ group including 34 patients ( 28 men and 6 women). There was no significant 
difference in gender, age and educational level among the groups, and thus the cases were comparable (Table I). The inclusion criterion was patients who met the diagnostic criteria for hepatitis $\mathrm{B}$ and cirrhosis of the liver according to consensus recommendations of the Asian Pacific Association for the Study of the Liver in 2009 (6). The exclusion criteria were as follows: Patients with evidence of mental illness, serious diabetes, thyroid disorders or other endocrine system and cardiovascular system diseases; having a history of drug use or drug addiction and alcohol dependence; pregnancy or lactation; appearing to be affected by the third stage of hepatic encephalopathy; unwilling to cooperate following communication with the medical staff; history of psychiatric drug use in the previous 2 weeks or a family history of mental illness; co-infection with other viruses (hepatitis A, E or C, or HIV); other causes of liver cirrhosis (Wilson's disease, other hereditary metabolic liver disease or autoimmune liver disease); co-existing hepatocellular carcinoma; portal vein thrombosis; renal impairment; or serious diseases in other organ systems. As there is a lack of liver donors, liver transplantation was not used regularly in this study. Therefore, patients who underwent liver transplantation were also excluded.

The study was performed according to the World Medical Association Declaration of Helsinki, and the protocol was approved by the medical ethics committee of the Third Affiliated Hospital of Sun Yat-Sen University. Written informed consent was obtained from all individuals included in this study.

General management of patients. The 114 patients were given standard comprehensive medical treatment (7) in accordance with the Asia-Pacific consensus recommendations, including absolute bed rest, intravenous antibiotics if required, a high calorie diet (35-40 cal/kg/day), lactulose, bowel enemas, maintenance water, electrolyte and acid-base equilibration, prevention and treatment of complications, and intensive care monitoring. Patients also received albumin, terlipressin, antiviral therapies (antiviral treatment including lamivudine, adefovir dipivoxil, telbivudine and entecavir were prescribed to the patients in whom hepatitis B virus replication was activated). The start date of the antiviral treatment was the date of diagnosis of hepatitis B cirrhosis among 58 patients, and 56 patients received antiviral therapy 2 years prior to the diagnosis of hepatitis B cirrhosis. There were 9 patients with viral breakthrough who were treated with antiviral drugs owing to unauthorized withdrawal prior to hospitalization, and proton pump inhibitors were administered or plasma exchange was performed if required. Orthotopic liver transplantation was not adopted, mainly due to the cost and lack of available donors.

Evaluation of psychiatric characteristics. The psychiatric characteristics of 114 cases of hepatitis B with cirrhosis were assessed using the HAMA $(8)$ and HAMD $(9,10)$. The HAMD is a multiple-choice questionnaire that clinicians use to rate the severity of a patient's depression. A score of 0-7 is considered to be normal, a score of $\geq 8$ is considered to be a mood disorder and a score of $\geq 20$ indicates moderately severe depression. The HAMA is a questionnaire used by clinicians to rate the severity of a patient's anxiety. It contains 14 symptom-oriented questions. Each of these symptoms is given a severity rating ranging from not present (score 0) to very severe (score 4 ). Total scores of $0-17$ are considered to indicate mild anxiety, those 18-25 mild to moderate anxiety, and those 26-30 moderate to severe anxiety. Each scale was jointly inspected and assessed by two staff who had received formal training. They scored individually, and consistency reached $\geq 90 \%$. If third-stage hepatic encephalopathy occurred or the patient succumbed in the process of treatment, the case was removed.

Statistical analysis. Continuous values are expressed as the mean \pm standard deviation and categorical values are presented as count and proportions. Between-group comparisons involving categorical data were performed using the $\chi^{2}$ statistic corrected for continuity; between-group comparisons involving continuous data (age and socio-economic status) were calculated using one-way analysis of variance and multiple comparisons. Data analysis was performed using SPSS software, version 16.0 (SPSS, Inc., Chicago, IL, USA). For all analyses, $\mathrm{P}<0.05$ was considered to indicate a statistically significant result.

\section{Results}

Baseline characteristics. In total, 114 patients with hepatitis B and liver cirrhosis were studied. Baseline characteristics including gender, educational level and basic information associated with liver and kidney function were similar in every patient group (Table I). There were found to be no significant differences in various baseline characteristics among the three groups $(\mathrm{P}>0.05)$ as presented in detail in Table I.

HAMA and HAMD scores of patients with hepatitis $B$ and cirrhosis. Following psychological evaluation by HAMD and HAMA, among the 114 cases of hepatitis B-associated cirrhosis of the liver in outpatient and hospitalized patients, there were 76 patients without mood disorders, accounting for $66.7 \%$ and 38 patients with mood disorders, accounting for $33.3 \%$. The results showed that the HAMA and HAMD scores in the CPG-C group were significantly higher than those in the CPG-A group $(\mathrm{P}<0.01)$, but not significantly higher than those in the CPG-B group $(\mathrm{P}>0.05)$. The proportion of patients with mood disorders in the $\mathrm{CPG}-\mathrm{C}$ group was significantly higher than that in the CPG-B group and CPG-A groups $(\mathrm{P}<0.05$ and $\mathrm{P}<0.01$, respectively; Table II).

Incidence of mood disorders in patients with hepatitis $B$ and cirrhosis according to disease severity. There were 76 cases without mood disorders, including 32 cases in the CPG-A group, 26 cases in the CPG-B group and 18 cases in the CPG-C group, accounting for 76.19, 68.42 and $52.94 \%$ of the respective CPG group. Among the 38 cases with mood disorders, the CPG-A group had 10 cases, the CPG-B group had 12 cases and the CPG-C group had 16 cases, accounting for $26.32,31.57$ and $42.11 \%$ of the cases with mood disorders, respectively. The prevalence of mood disorders in the CPG-C group of cirrhotic hepatitis B patients was clearly higher 
Table I. Baseline characteristics of included patients at admission.

\begin{tabular}{lccc}
\hline Characteristic & Child-Pugh A $(\mathrm{n}=42)$ & Child-Pugh B $(\mathrm{n}=38)$ & Child-Pugh C (n=34) \\
\hline Age & $41.6 \pm 9.7$ & $42.75 \pm 10.37$ & $42.79 \pm 11.2$ \\
Gender (male/female) & $35 / 7$ & $32 / 6$ & $28 / 6$ \\
Educational level, n $(\%)$ & & & $11(32.35)$ \\
Primary school & $13(30.95)$ & $11(28.95)$ & $22(64.71)$ \\
Middle school & $26(61.91)$ & $25(65.79)$ & $1(2.94)$ \\
College & $3(7.14)$ & $2(5.26)$ & $38.6 \pm 7.8$ \\
ALT & $39.4 \pm 8.5$ & $42.4 \pm 9.2$ & $25.95 \pm 10.32$ \\
TBil $(\mu \mathrm{mol} / \mathrm{l})$ & $19.73 \pm 9.25$ & $24.43 \pm 9.80$ & $67.35 \pm 39.38$ \\
SCR $(\mu \mathrm{mol} / \mathrm{l})$ & $69.53 \pm 35.36$ & $66.49 \pm 45.27$ & \\
\hline
\end{tabular}

Values are presented as the mean standard deviation, unless otherwise indicated. $\mathrm{P}>0.05$ for all intergroup comparisons of all characteristics. ALT, alanine transaminase; TBil, total bilirubin; SCR, serum creatinine.

Table II. HAMA and HAMD scores and the incidence of mood disorders.

\begin{tabular}{|c|c|c|c|}
\hline Rating scale & Child-Pugh A (n=42) & Child-Pugh B $(\mathrm{n}=38)$ & Child-Pugh C $(n=34)$ \\
\hline \multicolumn{4}{|l|}{ HAMA } \\
\hline Physical & $9.70 \pm 3.12^{\mathrm{a}}$ & $11.32 \pm 2.36^{\mathrm{b}}$ & $13.43 \pm 3.16^{\mathrm{c}}$ \\
\hline Mood & $13.43 \pm 2.86^{\mathrm{a}}$ & $16.51 \pm 2.99^{\mathrm{b}}$ & $21.26 \pm 3.59^{c}$ \\
\hline Anxiety & $10.22 \pm 3.23^{\mathrm{a}}$ & $13.12 \pm 4.33^{\mathrm{b}}$ & $15.12 \pm 5.13^{\mathrm{c}}$ \\
\hline Weight & $1.11 \pm 0.09^{\mathrm{a}}$ & $1.41 \pm 0.09^{\mathrm{b}}$ & $1.91 \pm 0.29^{c}$ \\
\hline Cognitive problems & $3.21 \pm 0.74^{\mathrm{a}}$ & $3.41 \pm 0.83^{b}$ & $3.82 \pm 0.95^{\mathrm{c}}$ \\
\hline \multicolumn{4}{|l|}{ HAMD } \\
\hline Diurnal variation & $0.33 \pm 0.028^{\mathrm{a}}$ & $0.35 \pm 0.03^{b}$ & $0.38 \pm 0.034^{\mathrm{c}}$ \\
\hline Lethargy & $8.06 \pm 2.04^{\mathrm{a}}$ & $8.26 \pm 2.13^{b}$ & $8.56 \pm 3.34^{\mathrm{c}}$ \\
\hline Sleep disorder & $4.85 \pm 1.10^{\mathrm{a}}$ & $5.06 \pm 1.31^{\mathrm{b}}$ & $6.06 \pm 1.31^{\mathrm{c}}$ \\
\hline Incidence of mood disorder, $\mathrm{n}(\%)$ & $10(23.81)^{\mathrm{a}}$ & $12(31.58)^{\mathrm{d}}$ & $16(47.06)^{\mathrm{c}}$ \\
\hline
\end{tabular}

Values are presented as the mean standard deviation, unless otherwise indicated. HAMA, Hamilton Anxiety Scale; HAMD, Hamilton Depression Scale. ${ }^{a} \mathrm{P}>0.05$ vs. Child-Pugh B; ${ }^{\mathrm{b}} \mathrm{P}>0.05$ vs. Child-Pugh $\mathrm{C} ;{ }^{\mathrm{c}} \mathrm{P}<0.01$ vs. Child-Pugh A; ${ }^{\mathrm{d}} \mathrm{P}<0.05$ vs. Child-Pugh C.

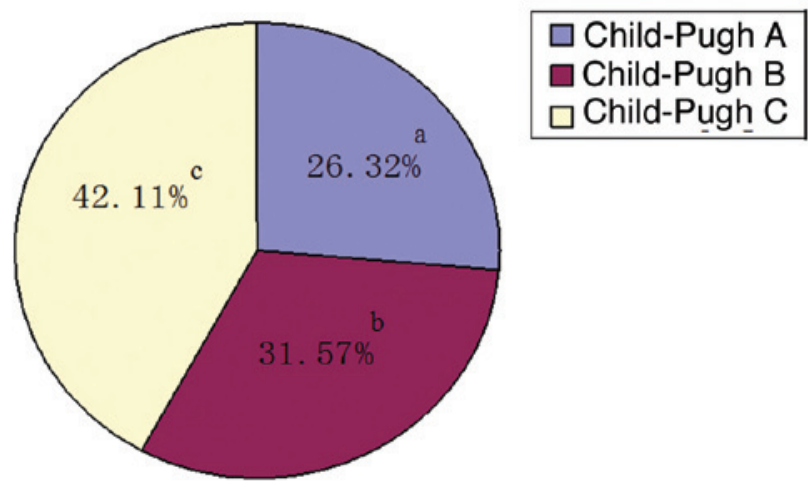

Figure 1.Percentages of the three Child-Pugh grades among the 38 patients with hepatitis B cirrhosis and mood disorders. ${ }^{a} \mathrm{P}<0.01$ vs. Child-Pugh $\mathrm{C} ;{ }^{b} \mathrm{P}>0.05$ vs. Child-Pugh $\mathrm{A} ;{ }^{\mathrm{c}} \mathrm{P}<0.05$ vs. Child-Pugh B.

than that in the CPG-A and CPG-B groups $(\mathrm{P}=0.0336$ for the latter). The proportion of patients with mood disorders was higher in the CPG-B group than in the CPG-A group, but the difference was not statistically significant $(P=0.4370)$. The rate of mood disorders in the $\mathrm{CPG}-\mathrm{A}$ group was significantly lower than that in the $\mathrm{CPG}-\mathrm{C}$ group $(\mathrm{P}=0.0078)$. The incidence of mood disorders in the three groups is presented in Fig. 1.

\section{Discussion}

Hepatitis B is one of the most prevalent infectious diseases in China (1). It is strongly contagious with long duration, no special treatment and a high cost of treatment $(1,11)$. When CHB develops into liver cirrhosis, it becomes an end-stage liver disease. Chronic liver disease has the characteristics of long duration and poor prognosis and it is harmful to patients' psychosomatic health. Furthermore, when a patient with an end-stage liver disease has a mental health problem, it is frequently misdiagnosed as a complication of hepatic encephalopathy, which results in ineffective treatments being administered and delays the diagnosis and treatment of the emotional disorder (9). There are some patients with 
end-stage liver disease who are suicidally depressed (10). Therefore, the psychological problems affecting patients with hepatitis B-associated liver cirrhosis require greater clinical attention.

The new biopsychosocial medical model encourages a greater focus of attention on the effect of psychological factors on physical disease. In particular, emotional disorders accompanying chronic disease, especially depression, have an apparent adverse influence on the treatment and prognosis of chronic disease. However, doctors typically ignore the diagnosis and treatment of depression when diagnosing physical diseases. This may owe to certain doctors habitually focusing on the diagnosis of physical disease and lacking knowledge of depression; in addition, the symptoms of depression can be covert and overlap with the symptoms of physical diseases (12).

Anxiety and depression are protective reactions produced and experienced by humans. Studies have indicated that psychological barriers clearly exist in patients with CHB, particularly anxiety and depression. Anxiety and depression are important factors that influence the clinical course of the disease and rehabilitation, and the occurrence of negative emotions in CHB cases is evident $(3,13)$.

The current treatments for hepatitis B are unable to eliminate the hepatitis virus from the body completely (14), which often causes patients to feel depressed about the incurable nature of the disease. Patients often worry excessively about their prognosis, which brings enormous psychological pressure and anxiety; in addition to the economic pressures of long-term treatment, patients are also concerned about the progress and infectivity of the disease. Furthermore, misunderstandings of the infectivity of hepatitis B and discrimination in society also increase the psychological pressure on patients with hepatitis B and liver cirrhosis, thus promoting the development of mental disorders or depression $(3,4,15)$. This is more apparent in patients with end-stage liver disease, which results from its long disease course and high case fatality rate, and the majority of patients feels down to various degrees and may experience depression (16). Therefore, the evaluation and judgment of depression in patients with end-stage liver disease is key to improving patients' confidence in conquering the disease and may be combined with other comprehensive therapies in order to improve the curative effect.

According to the results of the present study, there was no significant difference in the baseline data, such as gender, age, educational level, and liver and kidney function, among the groups, ruling out any interference due to inter-group differences. There were 38 patients in the study that also had mood disorders, which accounted for an incidence of mood disorders of approximately one-third. This result indicates that in addition to physical illness, a certain proportion of patients with hepatitis B and cirrhosis also have mood disorders, and indirectly implies that mood disorders develop in such patients not only because of hepatic encephalopathy but also because of the disease itself. These results are consistent with the findings of Song et al (13). The HAMA and HAMD scale scores of the patients with hepatitis B and cirrhosis in the present study had a tendency to increase as the severity of the hepatitis B-associated cirrhotic liver disease increased.
It may be hypothesized that this is due to the severity of the liver disease increasing or the long course of the disease. However, there was only a statistically significant difference in HAMA and HAMD scale scores between the patients in the CPG-A and CPG-C groups, which is consistent with the results of previous studies $(17,18)$. Mood disorders were identified in $23.81 \%$ of patients in the CPG-A group, $31.58 \%$ of the CPG-B group and $47.06 \%$ of the CPG-C group. The proportion of patients affected by mood disorders increased as the severity of the disease worsened. Compared with the CPG-A group, the incidence of mood disorder in the CPG-C group was increased significantly. These results were consistent with the HAMA and HAMD scale scores. It may be hypothesized that the incidence of depression increases as the illness is exacerbated because the treatments for hepatitis B-induced cirrhosis do not have a total curative effect; this increases depression and causes patients to lose hope (19). This is associated with the disease being chronic and difficult to treat, and the patients becoming anxious and confused regarding the long-term treatment and recurring effects. Therefore, it is recommended that for patients who experience such emotions, in addition to treating the liver disease, psychological care should also be strengthened.

In conclusion, in patients with hepatitis B-associated liver cirrhosis, depression is closely associated with the severity of the cirrhosis; patients with end-stage liver disease in particular, have a higher incidence of depression. It is recommended that importance should be attached to the assessment and treatment of emotional disorders when positively protecting the liver with comprehensive medical treatment. The results of this study should help to improve the success rate of the treatment of end-stage liver disease, for decompensated hepatitis B-associated liver cirrhosis in particular, and improve the quality of life patients with this disease.

\section{Acknowledgements}

The present study was supported by the Science and Technology project (grant no. 2014A020212059) and the Natural Science Fund (grant no. 2015A030313172) of Guangdong province, the Guangzhou city Science and Technology project entitled 'Receptor interacting protein-3 (RIP3) in the early warning role of aggravation of acute-on-chronic hepatitis B liver failure', and the Guangzhou city Science and Technology project (grant no. 1561000155). The authors thank all staff members of the Department Of Infectious Diseases of the Third Hospital of Sun Yat-Sen University.

\section{References}

1. Cornberg M, Jaroszewicz J, Manns MP and Wedemeyer H: Treatment of chronic hepatitis B. Minerva Gastroenterol Dietol 56: 451-465, 2010.

2. Wiersma ST, McMahon B, Pawlotsky JM, Thio CL, Thursz M, Lim SG, Ocama P, Esmat G, Mendy M, Bell D, et al: Treatment of chronic hepatitis B virus infection in resource-constrained settings: Expert panel consensus. Liver Int 31: 755-761, 2011.

3. Shen CZ, Shao AX, Ni JP, Zheng XH and Wang XY: Analysis on psychological status of patients with chronic hepatitis B. Hulixue Zazhi 19: 11-13, 2004 (In Chinese).

4. Chen Q, Jin GX and He ZB: Analysis on the depressive state of the patients with chronic hepatitis and nursing care. Hulixue Zazhi 16: 466-468, 2001 (In Chinese). 
5. Sarin SK, Kumar A, Almeida JA, Chawla YK, Fan ST, Garg H, de Silva HJ, Hamid SS, Jalan R, Komolmit P, et al: Acute-on-chronic liver failure: Consensus recommendations of the Asian Pacific Association for the Study of the Liver (APASL). Hepatol Int 3: 269-282, 2009.

6. Soga K, Tomikashi K, Miyawaki K, Fukumoto K, Wakabayashi N, Konishi H, Mitsufuji S, Yoshida N, Kokura S, Naito Y, et al: MELD score, Child-Pugh score and decreased albumin as risk factors for gastric variceal bleeding. Hepatogastroenterology 56 : $1552-1556,2009$.

7. Zheng YB, Xie SB, Xie DY, Peng L, Lei ZY, Deng H, Lin BL Lin CS, Zhao ZX, Ke WM and Gao ZL: A Novel prognostic scoring system to predict 3-month mortality risk in patients with acute-on-chronic liver failure in hepatitis B: A retrospective cohort study. Hepatol Int 6: 727-734, 2012.

8. Hamilton M: The assessment of anxiety states by rating. Br J Med Psychol 32: 50-55, 1959.

9. Hamilton M: A rating scale for depression. J Neurol Neurosurg Psychiatry 23: 56-62, 1960

10. Mowla A, Kalantarhormozi MR and Khazraee S: Clinical characteristics of patients with major depressive disorder with and without hypothyroidism: A comparative study. J Psychiatr Pract 17: 67-71,2011.

11. Pan C, Gu Y, Zhang W, Zheng Y, Peng L, Deng H, Chen Y Chen L, Chen S, Zhang M and Gao Z: Dynamic changes of lipopolysaccharide levels in different phases of acute on chronic hepatitis B liver failure. PLoS One 7: e49460, 2012.
12. Zhang M: Diagnosis and treatment of psychologic disorders in outpatients in general hospitals. Zhonghua Yi Xue Za Zhi 79: 325-326, 1999 (In Chinese).

13. Song JX, Liu XD, Qi JY and Tian DY: The survey of psychiatric disorders among the patients with chronic viral hepatitis and treatment measures. Tong Ji Yi Ke Da Xue Xue Bao 30: 266-268, 2001 (In Chinese).

14. Fan ZP, Wang FS, Xu DP, Chu FL, Shi M, Zhou Y and Zhang LX: Detection of HBcAg-specific cytotoxic lymphocytes and their association with clinical status in patients with hepatitis B. Zhonghua Yi Xue Za Zhi 84: 2073-2076, 2004 (In Chinese).

15. Tu B and Shao W: Chronic hepatitis B with depression treatment efficacy ademetionine. Zhong Xi Yi Jie He Gan Bing Za Zhi 19: 174-175, 2009 (In Chinese).

16. Novelli G, Rossi M, Ferretti G, Pugliese F, Ruberto F, Lai Q, Novelli S, Piemonte V, Turchetti L, Morabito V, et al: Predictive criteria for the outcome of patients with acute liver failure treated with the albumin dialysis molecular adsorbent recirculating system. Ther Apher Dial 13: 404-412, 2009.

17. Lu MY and Zheng DR: The psychological care of the patients with chronic hepatitis B. Zhongguo Yiyao Daobao 8: 192-193, 2011 (In Chinese).

18. Xu HY: Advances in the treatment of depression in chronic liver medicine. Beijing Zhong Yi 32: 9-10, 2013 (In Chinese).

19. Li XQ, Cai X, Pei B, Chen CS and Xu Z: Investigation on mental status of chronic hepatitis B patients. Di 4 Jun Yi Da Xue Xue Bao 28: 1802-1804, 2007 (In Chinese). 